Scientific Visualization, 2019, volume 11, number 4, pages 130 - 139, DOI: 10.26583/sv.11.4.11

\title{
Optical diagnostics of temperature field of an axisymmetric flame
}

\author{
Yu.N. Dubnishcheve,A,C,D, V.A. Arbuzov,A,C, E.V. Arbuzov3,B,C, \\ O.S. Zolotukhina ${ }^{4, C}$, V.V. Lukashov5,A \\ A Kutateladze Institute of Thermophysics, \\ Siberian Branch of the Russian Academy of Sciences \\ B Sobolev Institute of Mathematics of the Siberian Branch of the \\ Russian Academy of Sciences \\ c Novosibirsk State Technical University \\ D Technological Design Institute of Scientific Instrument Engineering of the Siberian \\ Branch of the Russian Academy of Sciences \\ ${ }^{1}$ ORCID: 0000-0001-7874-039X, dubnistchev@itp.nsc.ru \\ 2 ORCID: 0000-0003-2404-326X, arbuzov@itp.nsc.ru \\ 3 ORCID: 0000-0001-9488-8650, arbuzov@math.nsc.ru \\ 4 ORCID: oooo-ooo3-3486-4459, melexinaolga17@yandex.ru \\ 5 ORCID: 0000-0001-8178-7607, luka@itp.nsc.ru
}

\begin{abstract}
The structure of the temperature field of an axisymmetric flame using an example of a candle, spiritlamp, and propane-air torch was studied. The optical diagnostics adapted to the study of combustion problems is based on the visualization by methods of Hilbert-optics of phase disturbances induced by the medium under study in a probe light field. The diagnostic complex is implemented on the basis of the IAB-463M device with modified blocks of optical filtration, light source, registration and information processing. The dynamic phase structure of the candle flame and spiritlamp was visualized as a classic object for approbation the diagnostic method. The dynamic phase structure of the propane-air torch was investigated. The temperature was measured using thermocouples at the reference points. The phase function was restored on axisymmetric sections from the obtained hilbertograms, and the temperature field of flame was reconstructed using the inverse Abel transform.
\end{abstract}

Keywords: optical diagnostics of flames, propane-air flame, Hilbert-optics.

\section{Introduction}

Methods of Hilbert-optics and interferometry constitute one of the directions of unperturbing diagnostics of reacting jets and flames $[1,2]$. They are based on visualization and analysis of phase disturbances induced by the studied medium in the probing light field. In [3, 4] studied diffuse combustion jet of hydrogen, by methods the Hilbert-optics. An optical diagnostic complex based on the IAB-463M instrument [5] equipped with modified Hilbert filtering, interference, light source, Hilbert image registration and information processing modules was used. In [6] a method for estimating the temperature distribution in an asymmetric flame using high-contrast stereoscopic photography is described. Spectral reconstruction of temperature fields using pyrometry of color ratios and interferometric tomography is reported [7]. A jet flame of premixed propane-air mixtures has a wide range of applications in scientific research and practical applications, the successful development of which requires the de- 
velopment of optical diagnostic methods with the possibility of reconstruction of the spatial phase and temperature structure of the burning torch.

The adaptation of the methods of Hilbert-optics to the solution of diagnostic problems with the reconstruction of the spatial phase and temperature structure of the flame is the goal of this work. The studies motivated by the scientific and practical significance of the problem, which consists in the search for control methods of the structural and thermodynamic parameters of the torch [8].

\section{Research method}

The optical diagnostics complex was created on basis of the IAB-463M [5] device with modified for the experiments modules of the probing field source, of Hilbert filtering, registration and processing of the optical signal.

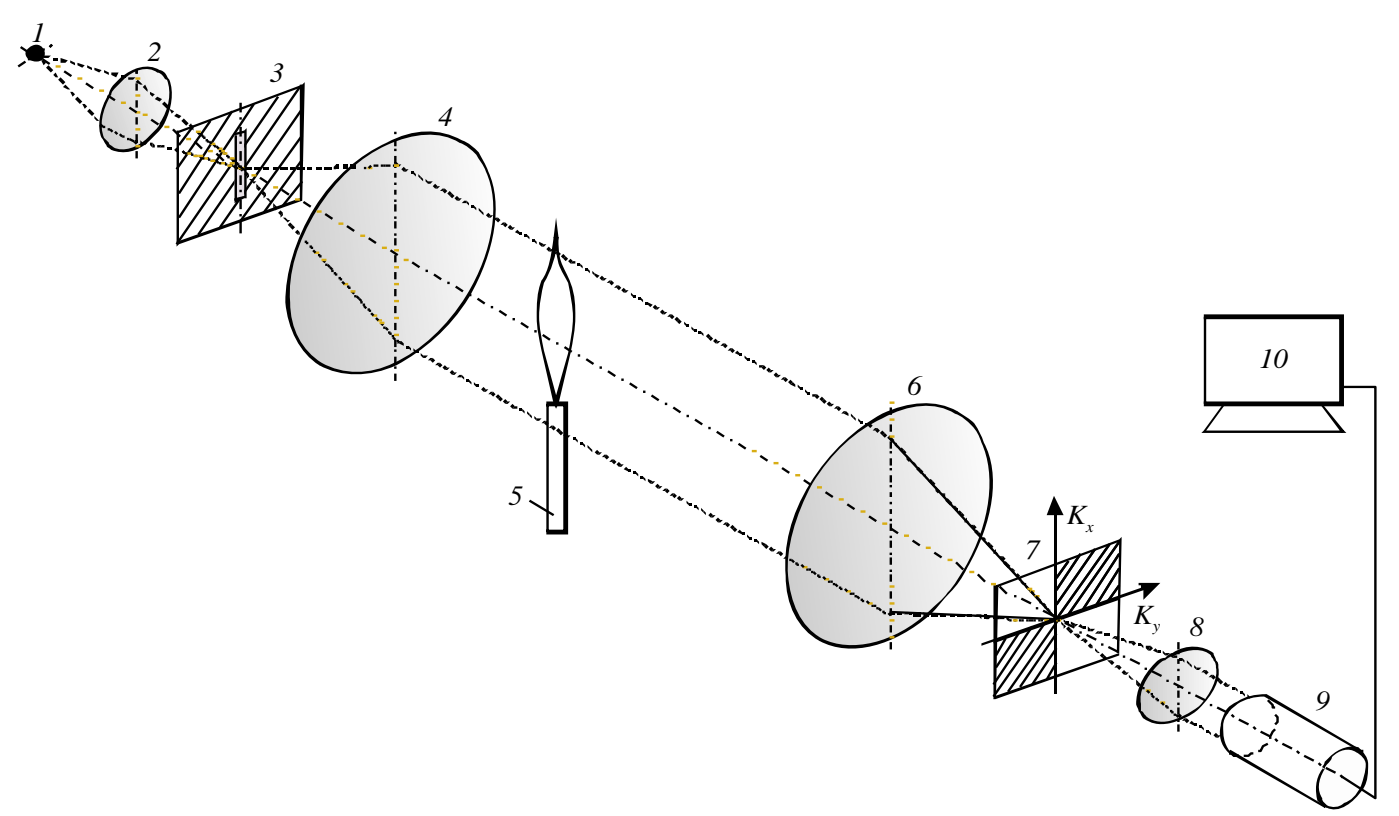

Fig.1. Optical diagnostics complex.

The optical flame diagnostics complex sketch is shown in Fig. 1. It contains a lighting module consisting of a light source 1, a lens 2, and a slit diafragm 3 located in the front Fourier plane of the lens 4, which forms a probe field. The Fourier spectrum of the phase disturbances induced by the medium under study 5 is localized in the frequency plane of the lens 6 , where the quadranted Hilbert-filter 7 is located, the orientation of which corresponds to the slit diafragm 3. The lens 8 performs the inverse Fourier transform of the filtered field, forming, depending on the spectral characteristics of the light source, analytical or Hilbert-conjugate optical signal. These signals are recorded by a digital camcorder 9 connected to the computer 10.

In the frequency plane $\left(K_{x}, K_{y}\right)$ for the Fourier spectrum of the phase optical density of the light field $s\left(K_{x}, K_{y}\right)$ perturbed by the medium (flame) under study, we have immediately after filter 7:

$$
H\left(K_{x}, K_{y}\right) s\left(K_{x}, K_{y}\right)=s\left(K_{x}, K_{y}\right) \cos \varphi+\hat{s}_{x}\left(K_{x}, K_{y}\right) \sin \varphi,
$$

where $H\left(K_{x}, K_{y}\right)$ is the transfer-function of the filter, $\hat{s}_{x}\left(K_{x}, K_{y}\right)$ is the Fourier spectrum of the Hilbert-conjugate optical signal:

$$
\begin{gathered}
H\left(K_{x}, K_{y}\right)=\cos \varphi-i \sin \varphi \operatorname{sgn} K_{x}, \\
\hat{s}_{x}\left(K_{x}, K_{y}\right)=-i \operatorname{sgn} K_{x} s\left(K_{x}, K_{x}\right) .
\end{gathered}
$$


Phase shift $\varphi$ is a function of wavelength $\lambda$ of the probe light field, $\varphi=\varphi(\lambda)$. The lens 8 performs the inverse Fourier transform of the filtered signal (1):

$$
s\left(K_{x}, K_{x}\right) \cos \varphi+\hat{s}_{x}\left(K_{x}, K_{x}\right) \sin \varphi \leftrightarrow s(x, y) \cos \varphi+\hat{s}_{x}(x, y) \sin \varphi .
$$

The recorded by the photo matrix of the camcorder 9 signal intensity (2):

$$
I(x, y)=\rho\left\{|s(x, y)|^{2} \cos ^{2} \varphi+\left|\hat{s}_{x}(x, y)\right|^{2} \sin ^{2} \varphi\right\},
$$

where $\rho$ is a coefficient taking into account the sensitivity of the photo matrix. The Fourier filter performs a one-dimensional Hilbert transform at a wavelength of $\lambda=\lambda_{0}$ satisfying condition $\varphi\left(\lambda_{0}\right)=\pi / 2$.

The Hilbert transform has the properties of redistributing energy from the region of low spatial frequencies to the high-frequency region. The extrema and gradients of the phase optical density of the medium under study transformed into visualized structures of the Hilbert bands. The spatial distribution of the Hilbert bands carries information on the perturbations of the phase optical density induced by the temperature field.

The flame of a candle and spirit lamp was chosen as a classic object for approbation the diagnostic method. Measurements of the temperature profile in flame sections at various distances from the end of the wick have been fulfilled using an Chromel-alumel thermocouple (Fig. 2). The Hilbert images of flames are shown in Fig. $3 \mathrm{a}$ and $3 \mathrm{~b}$.

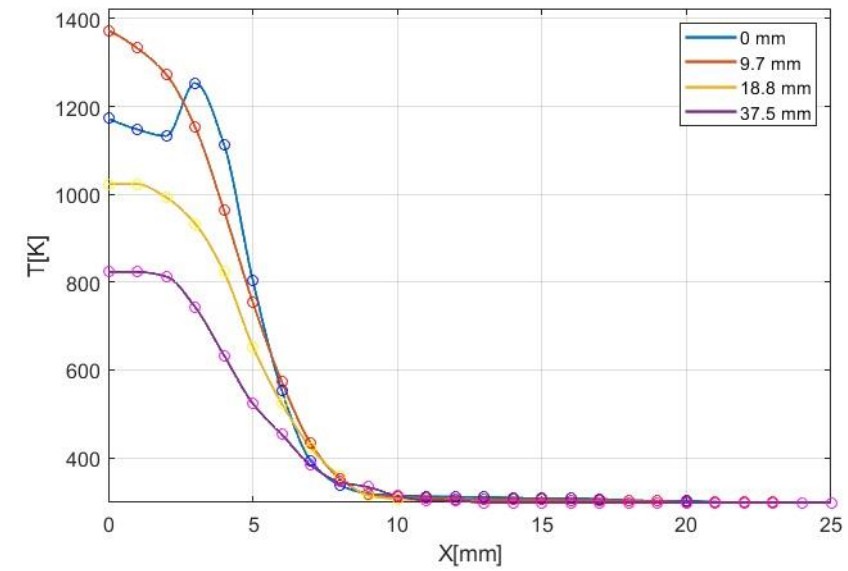

a)

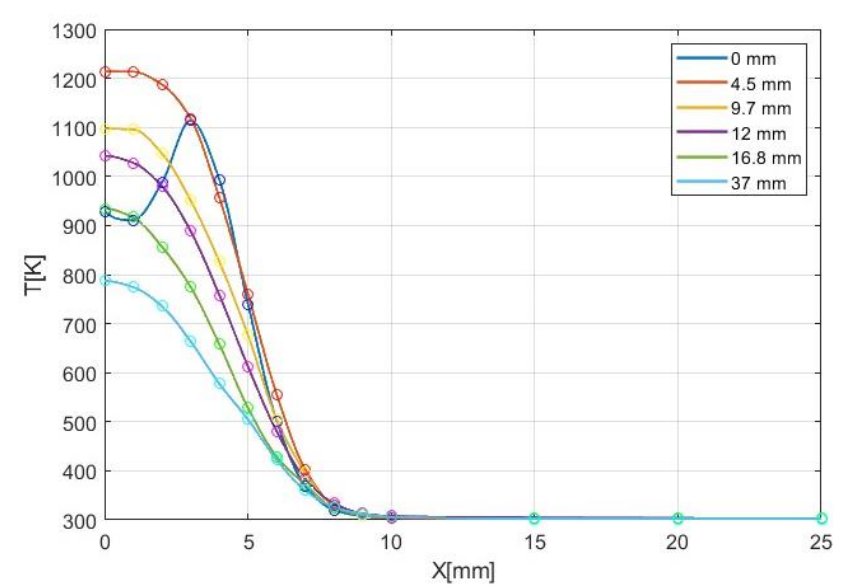

b)

Fig. 2. Temperature profiles of the flame: (a) - candles in cross sections at distances of $0 \div$ $37,5 \mathrm{~mm}$ from the wick; (b) - spirit lamps at distances of $0 \div 37 \mathrm{~mm}$ from the wick.

The phase structure of the probe light field disturbed by the medium under study is:

$$
\Delta \psi(x, y)=k \int_{z_{1}}^{z_{2}}\left[n(x, y, z)-n_{0}\right] d z,
$$

where $k=2 \pi / \lambda$ is the wave number of the probe field; $n(x, y, z)$ - refractive index of the medium in the spatial structure of the flame; $n_{0}$ - refractive index of an unperturbed medium. The $z$ axis is determined by the direction of the probe light beam, the flame torch cross section is described in $x, z$ coordinates. The choice of the section position is determined by the $y$ coordinate. The coordinates $z_{1}, z_{2}$ specify the size of the flame section in the direction of the probe beam.

Eq. (4) is turned into the Abel equation in the case of axial flame symmetry:

$$
\Delta \psi(x, y)=2 k \int_{x}^{R}\left[n(r)-n_{0}\right] \frac{r d r}{\sqrt{r^{2}-x^{2}}},
$$

where $r^{2}=x^{2}+y^{2}, R$ - the radius of the cross section of the considered zone, $n(r)$ - refractive index at a distance of $r$ from the axis of the torch. The Abel equation is solved by an approximate method based on approximation of the experimental temperature data by Bezier curves (a special case of B-splines). 


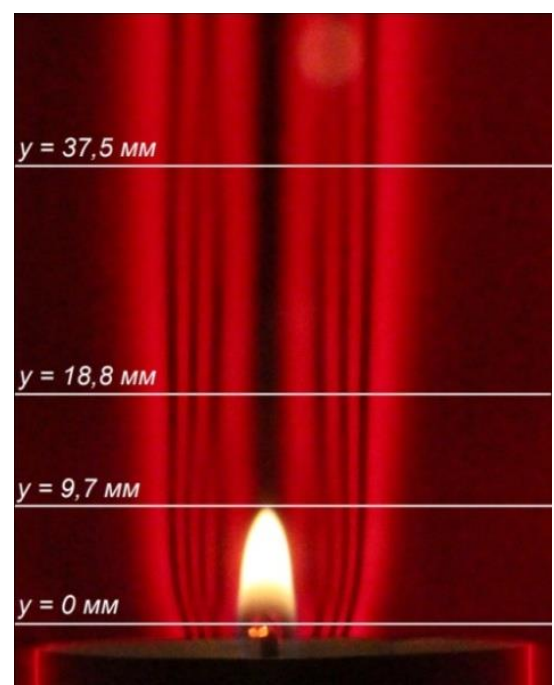

a)

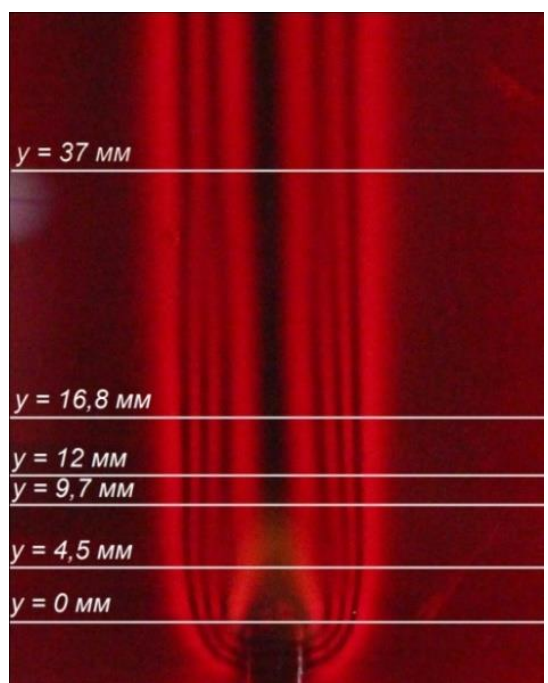

b)

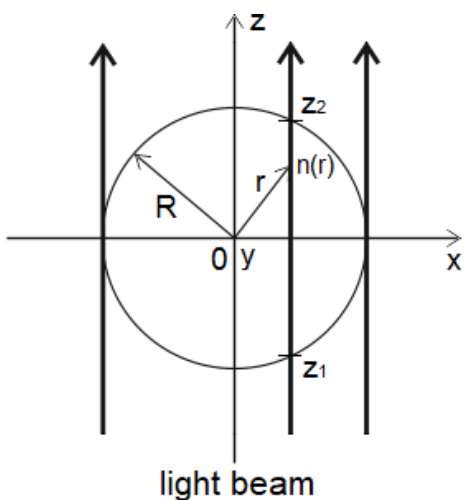

c)

Fig. 3. Experimental hilbertograms: (a) - the candle flame, (b) - the spirit lamp flame; (c) section diagram of the flame under study in the plane $y=$ const.

The total phase shift $\psi(x, y)$ for the light beam in section $y=$ const depends on the refractive index $n(r, x)$ on the segment $\left(z_{1}, z_{2}\right)$, (Fig.3c).

The axisymmetric distribution of the refractive index $n(r, y)$ found from the Abel equation allows one to determine the radial fields of temperature $T(r, y)$ in the cross sections $(y)$. To do this, can use the Gladstone - Dale equation:

$$
T(r, y)=\left[\frac{n_{0}-1}{n(r, y)-1}\right] T_{0},
$$

Graphs illustrating the restoration of the radial distribution of the temperature of the flame of a candle and an spirit lamp in sections $y=18,8 \mathrm{~mm}$ and $y=16,8 \mathrm{~mm}$ from the end of the wick using equations (5) and (6) are presented in Fig. 4. The red line (Fig. 4 (2)) shows the phase function $\psi(x, y)$ obtained from the Abel equation in the selected sections, the green and black lines represent the interferogram and the hilbertogram recoverded from the phase function $\psi(x, y)$. The blue line is the experimental hilbertogram.

A comparison of the experimental and reconstructed hilbertograms in the selected sections shows that the points of local minima of these functions coincide.This means that the phase function obtained from the solution of the Abel equation coincides with the real phase function. Therefore, the real and the reconstructed temperature fields in the selected flame sections coincide.The criterion for the correctness of the results obtained is the phase identity of the reconstructed and experimental hilbertograms, which is achieved by repeating the described procedure.
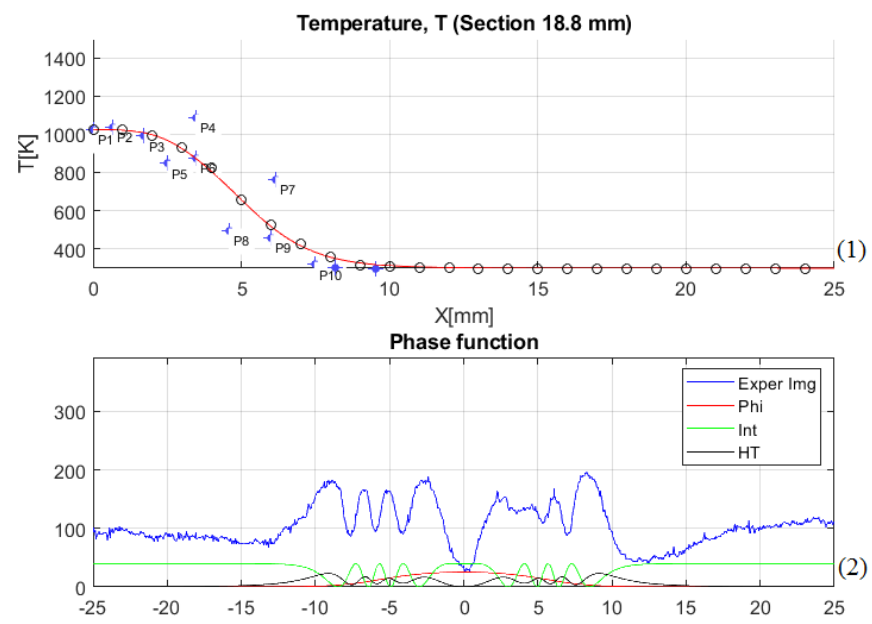

a) 

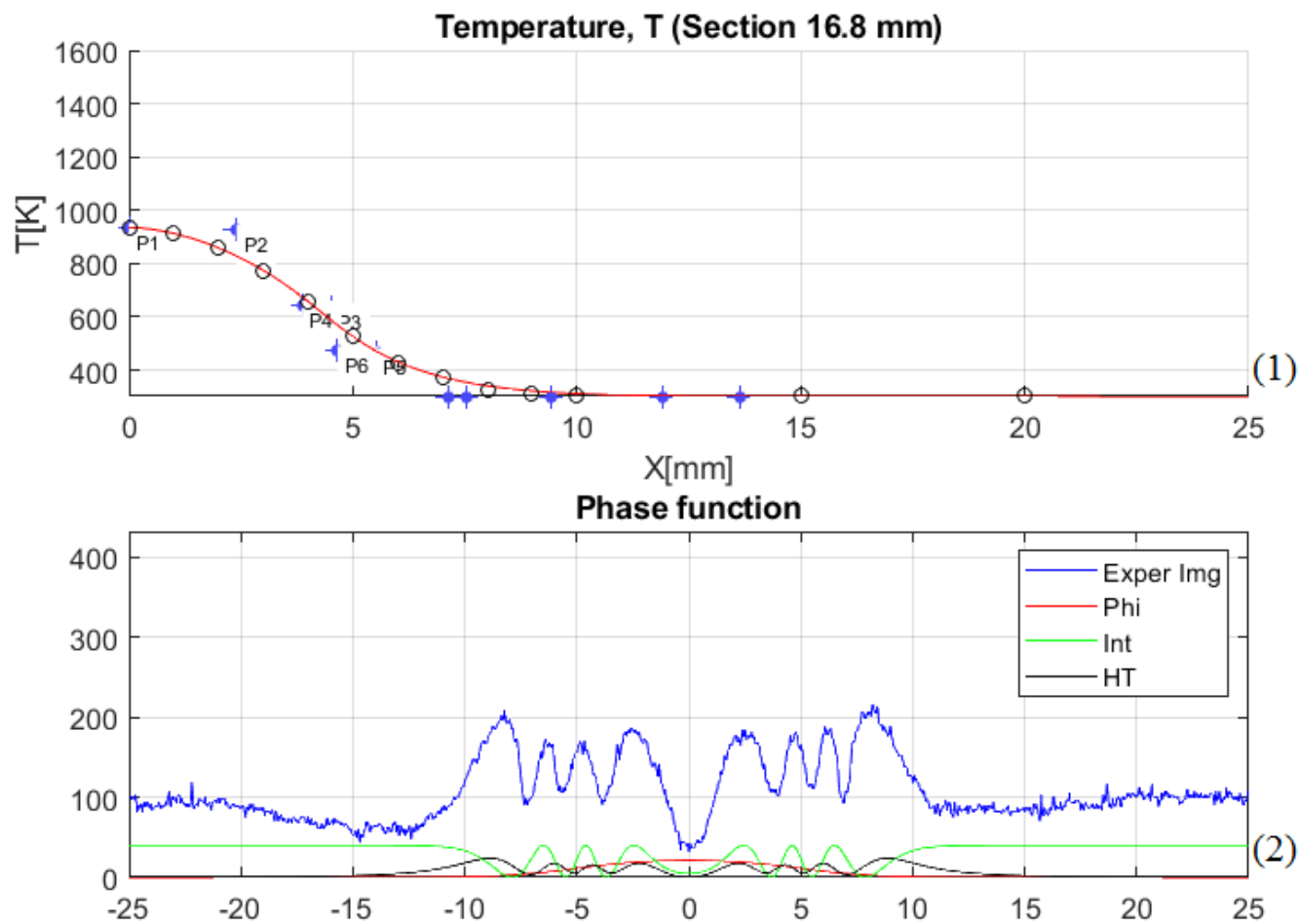

b)

Fig. 4. Reconstruction of the phase structure and temperature of the candle flame (a) and the spirit lamp (b) in the sections $y=18,8 \mathrm{~mm}$ and $y=16,8 \mathrm{~mm}$ : (1)

the red line - restored temperature (small circles - thermocouple data); (2) the red line phase function, the green line -interferogram reconstructed from the phase function, the black line - reconstructed hilbertogram, and the blue line - experimental hilbertogram.

The temperature fields reconstructed from the results of measurements using thermocouples are shown in Fig. 5a and 6a. The temperature fields reconstructed from the Hilbert images are shown in Fig. $5 \mathrm{~b}$ and $6 \mathrm{~b}$. The similarity of reconstructed and original temperature fields is quite satisfactory.

As a verification of the obtained results, the inverse problem has been solved: from the reconstructed temperature fields (Fig. 5b and 6b) have been restored Hilbert images (Fig. $7 \mathrm{~b}$ and 7d), which were compared to the images obtained in the experiment (Fig. 7a and 7c).

From Fig. 7 it can be seen that the structures obtained in the experiment and reconstructed have a similar character. By this confirms the reliability of the results. Some discrepancy is due to the distortion of the axial symmetry of the flame in a real experiment due to the influence of dynamic disturbances of the air environment that surrounding the flame.

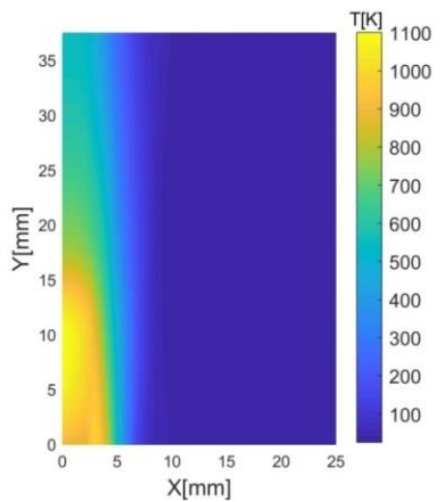

a)

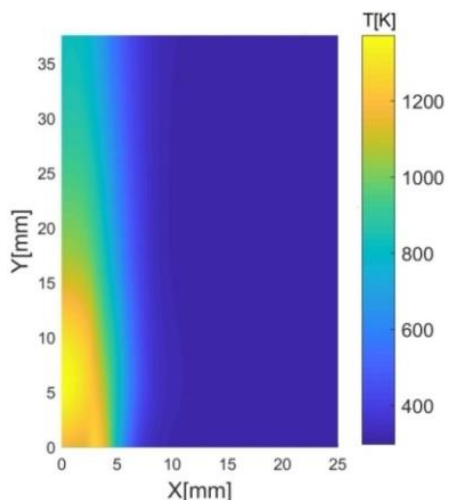

b)

Fig. 5. The temperature field of a candle flame: (a) - the reconstructed according to thermocouple data, (b) - the reconstructed from a Hilbert image. 


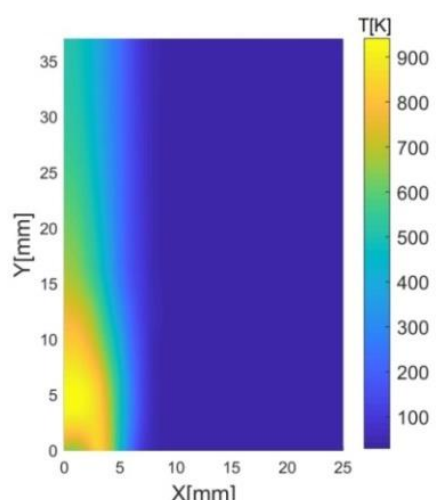

a)

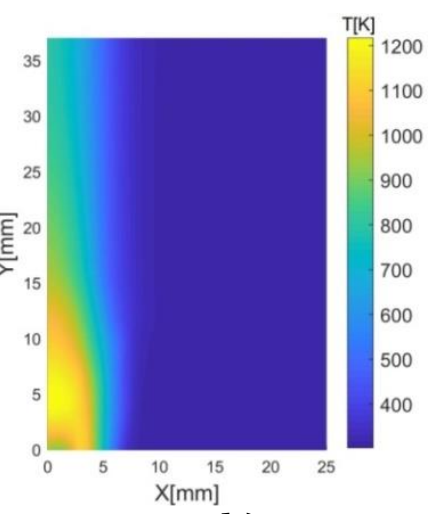

b)

Fig. 6. The temperature field of the spirit lamp flame: (a) - the reconstructed according to thermocouple data, (b) - the reconstructed from a Hilbert image.

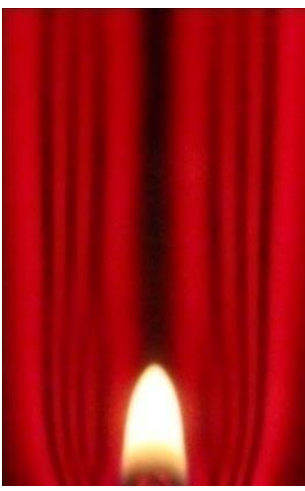

a)

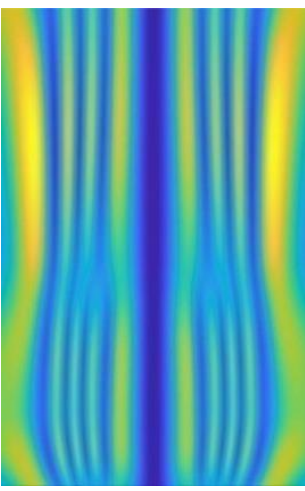

b)

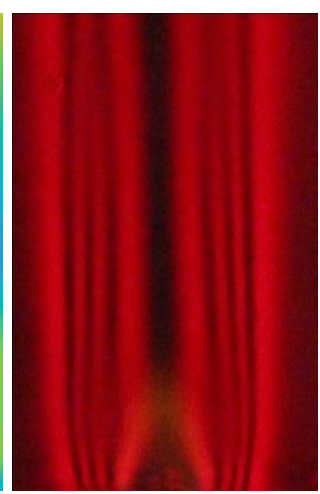

c)

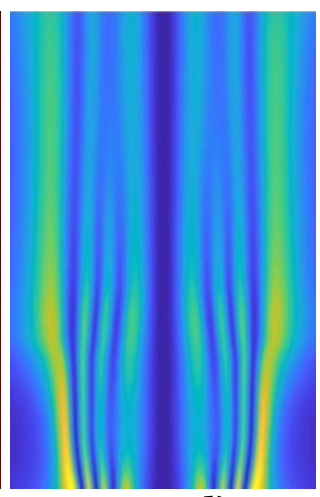

d)

Fig. 7. Flame of a candle and spirit lamp: (a) and (c) - the hilbertograms of the flame of a candle and spirit lamp obtained in the experiment; (b) and (d) - the hilbertograms of the flame of a candle and spirit lamp numerically modeled from the reconstructed temperature fields.

The developed method of optical diagnostics was successfully applied in studies on the jet combustion of premixed propane-air flame.

The Hilbert image of the jet combustion of a premixed propane-air mixture (propane concentration 25\%) in a still atmosphere (air) is shown in Fig. 8a. The gradient phase structure of the probe light field induced by the temperature field in the torch was visualized. The results of measuring the temperature profile of the flame in cross sections at distances of $0 \div 20 \mathrm{~mm}$ from the end of the tube are shown in Fig. 8b. The measurements were carried out using a TPR thermocouple. A chart illustrating the restoration of the radial temperature distribution in the flame cross section $y=20 \mathrm{~mm}$ from the end of the tube is shown in Fig. 8c.

Fig. 9a and $9 b-$ the temperature fields obtained from the data thermocouple measurements and reconstructed from the hilbertograms, respectively.

Fig. 10a and 1ob illustrate the comparison of Hilbert images of the reconstructed and experimentally obtained temperature fields. 


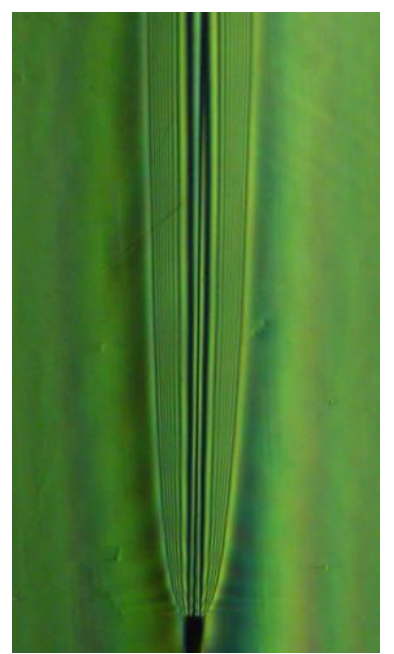

a)

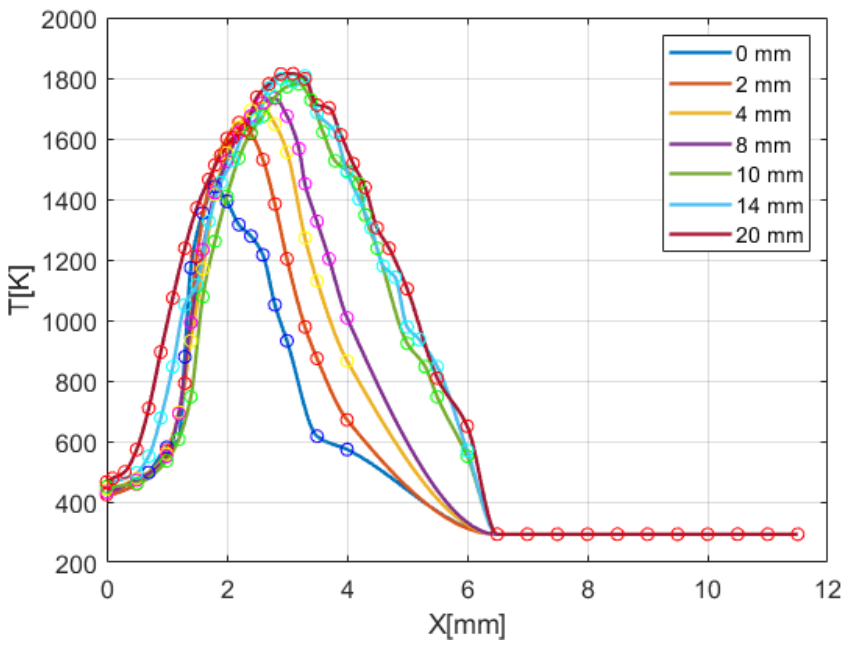

b)
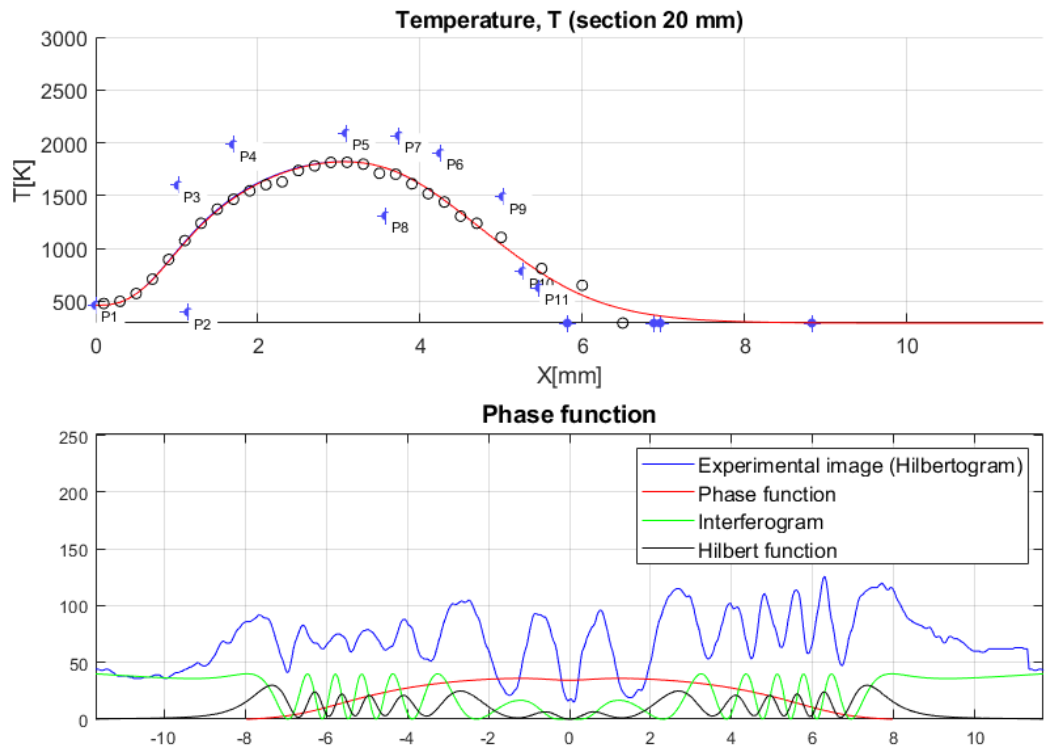

c)

Fig. 8. Propane-air flame: (a) -Hilbertogram of jet combustion of a premixed propane-air mixture of $25 \%$ in air; (b) - temperature profiles of jet combustion of a propane-air mixture of $25 \%$ at distances of $0 \div 20 \mathrm{~mm}$; (c) -reconstruction of the phase structure and torch temperature in the cross section $y=20 \mathrm{~mm}$.

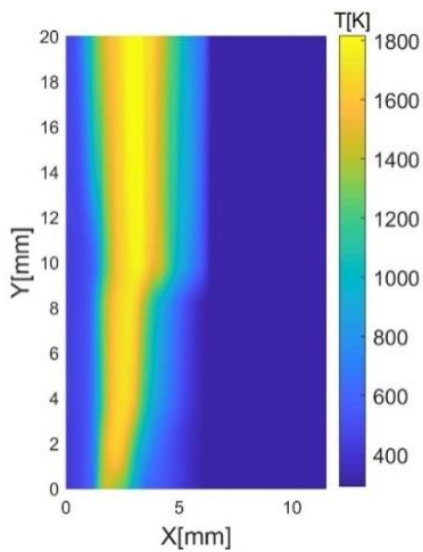

a)

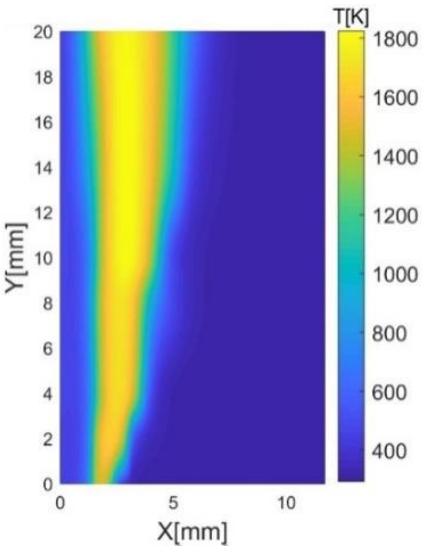

b)

Fig.9. Temperature field of the propane-air flame: (a) - the temperature field reconstructed from the thermocouple measurements, (b) - the temperature field reconstructed from the Hilbert image. 


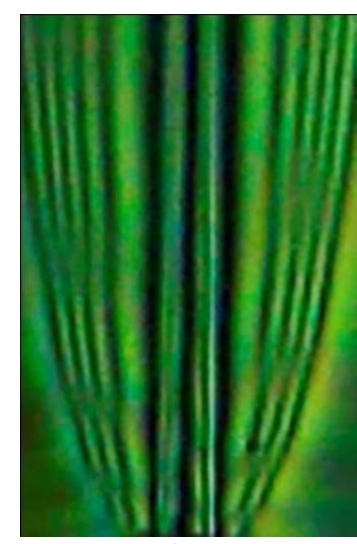

a)

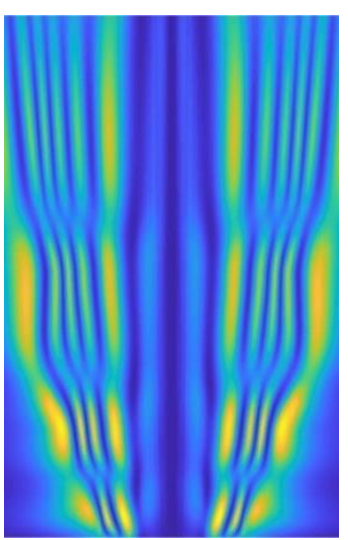

b)

Fig. 10. Hilbertograms of the propan-air torch: (a) - the hilbertogram obtained in the experiment; (b) - the hilbertogram numerically modeled from the reconstructed temperature field.

\section{Conclusion}

The study of the flame of a candle, spirit lamp and premixed propane-air torch was performed in the presented work. For this, the methods of Hilbert optics in the approximation of axial symmetry using the Abel transform were used. The reliability of the results is confirmed by comparing the hilbertograms obtained in the experiment and reconstructed from the phase structure according to Abel. The comparison results are used as a quality criterion in modeling of the phase structure and temperature field in the study of the combustion process. The authors thank N. S. Bufetov for help in the work.

This work was supported by the Ministry of Education and Science of the Russian Federation (AAAA-A17-117030310010-9) and by Siberian Branch of the Russian Academy of Sciences (complex program of fundamental scientific research SB RAS II.1 project 0314-2018-0010).

\section{References}

1. Dubnishchev Yu. N., Arbuzov V. A., Belousov P. P., Belousov P. Ya. Opticheskie metody issledovaniya potokov [Optical Methods of Flow Investigation]. Sib. Univ. Izd., Novosibirsk, 2003, 418 p. [in Russian]

2. Dubnishchev Yu. N. Teoriya i preobrazovanie signalov v opticheskih sistemah [Theory and Transformation of Signals in Optical Systems]. St.-Petersburg: Publishing house «Lan», 2011, 368 p. [in Russian]

3. 2.Dubnishchev Yu. N., Lemanov V. V., Lukashov V. V., Arbuzov V. A., Sharov K. A. Hydrodynamic vortexs tructure sinadiffusi on jet flame (electronic edition) // «Swirling Flows and Flames», pp. 1-21. DOI: 10.5772/intechopen.80610. https://www.intechopen.com/online-irst/hydrodynamic-vortex-structures-in-adiffusion-jet-flame. Published: November $5^{\text {th }} 2018$.

4. Yu. N. Dubnishchev, V. A. Arbuzov, V. V. Lukashov, K. A. Sharov, V. V. Lemanov. Optical Hilbert Diagnostics of Hydrogen Jet Burning// Optoelectron., Instrum. Data Process., 2019, 55: pp. 16 - 19. https://doi.org/10.3103/S8756699019010035

5. Belozerov A. F. Opticheskie metody vizualizacii potokov [Optical Methods of Gas Flow Visualization]. Izd. Kazan. Gos. Tekh. Univ., Kazan', 2007, 747 p. [in Russian]

6. Qunxing Huang, Fei Wang, Jianhua Yan, and Yong Chi. Simultaneous estimation of the 3D soot temperature and volume faction distributions in asymmetric flames using highspeed stereoscopics images // Applied Optics, 2012, 51 (15), pp. 2968 - 2978.

7. Jochen A. H. Dreyer, Radomir I. Slawchov, Eric J. Rees, Jethro Akroyd, Maurin Salamanca, Sebastian Mosbach, and Markus Kraft. Improved methodology for performing the in- 
verse Abel transform of flame images for cilir ratio pyrometry // Applied Optics, 2019, 58 (10), pp. $2662-2670$.

8. Yu. A. Litvinenko Yu. A. "Stability of Subsonic Macro- and Microjets and Microjet Burning (Review),” Sib. Fiz. Zh. 12 (3), 2017, pp. 83 - 99. 